\title{
Systems biology and metabolic networks predict heterosis
}

Heterosis or hybrid vigour is the improved function of a desirab quality in hybrid offspring. Predicting heterosis would mean that plant and animal time-consuming and costly field tests of numerous combinations. Dominique de Vienne, Professor at the University Paris-Saclay, France, uses metabolic

networks and systems biology to study heterosis. Modelling metabolic systems has relied on theoretical methods that require long-term effort from many pathways and do not work well for complex systems. Now a conceptual shortcut that can simulate complexity using fewer biological data has been found.

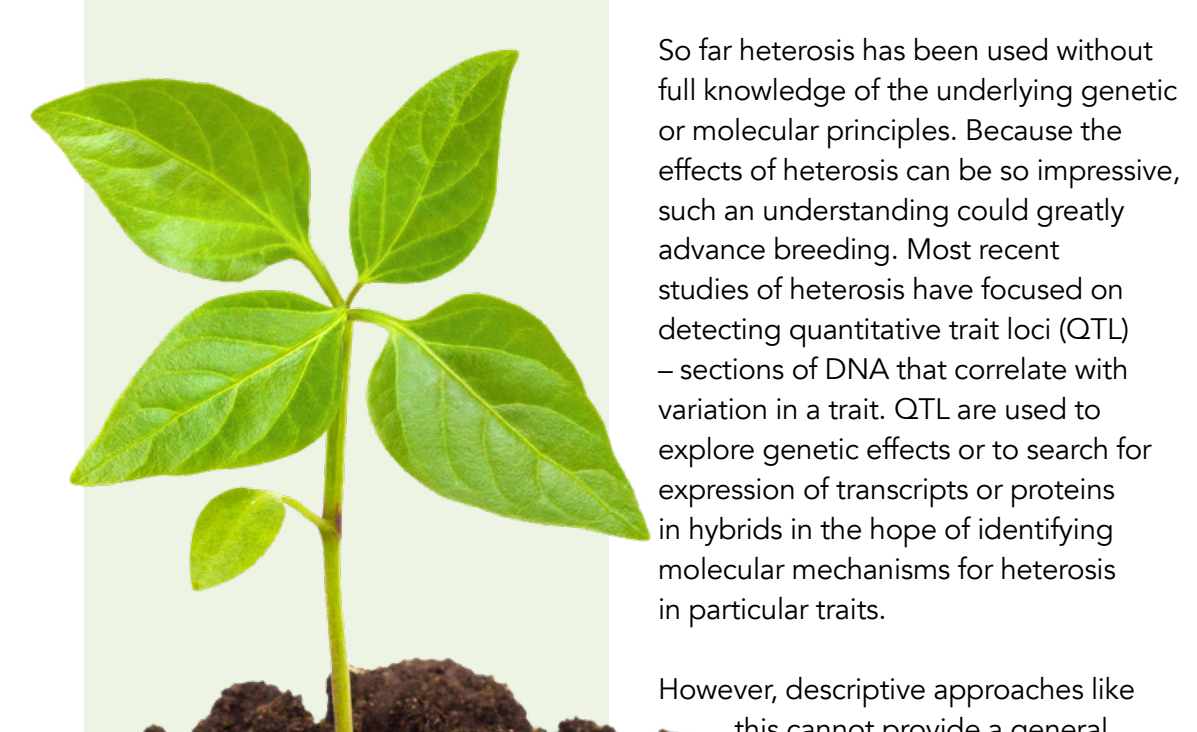

this cannot provide a genes like this cannot provide a general

model accounting for the pervasivenes of heterosis. This is where a systemic modelling is proving useful. vigour than their parents for quantitative traits. For example, growth is faster age of reproduction is earlier, fertility is higher and resistance to disease is heterosis, has been exploited by human in animal and plant breeding and has

The introduction of cross-pollinated (hybrid) plants was one of the most important innovations in agriculture and global food security. Most annual fops show heterosis. Hybrid maize, as the por can yield twice as much of some hybrid yeasts growth rate of parental strains by more than an order of magnitude.

\section{SYSTEMS AND NETWORK BIOLOGY} Heterosis has inspired many genetic,

has less often been investigated from the perspective of systems biology, Prof Dominique de Vienne's focus. Systems biologists model complex biological systems, such as molecules and their interactions within a living cell, rather than looking at isolated parts.

Related to this is network biology enabling the representation and analysis of biological systems with tools derived from graph theory, which uses mathematical structures objects, and topologions between the arrangement of the wichents of a network.

Network analysis works with the complexity of the network to extract meaningful information that you would not have if individual components were examined separately. The data explosion from the 'omics' era of biological research has led to more systemic approaches to data analysis and a move away from single gene/protein studies.

Adding the ending 'omics' to a molecular term implies a comprehensive Genomics, the first omics apules, Genomics, the first omics approach, focused on entire genomes as opposed

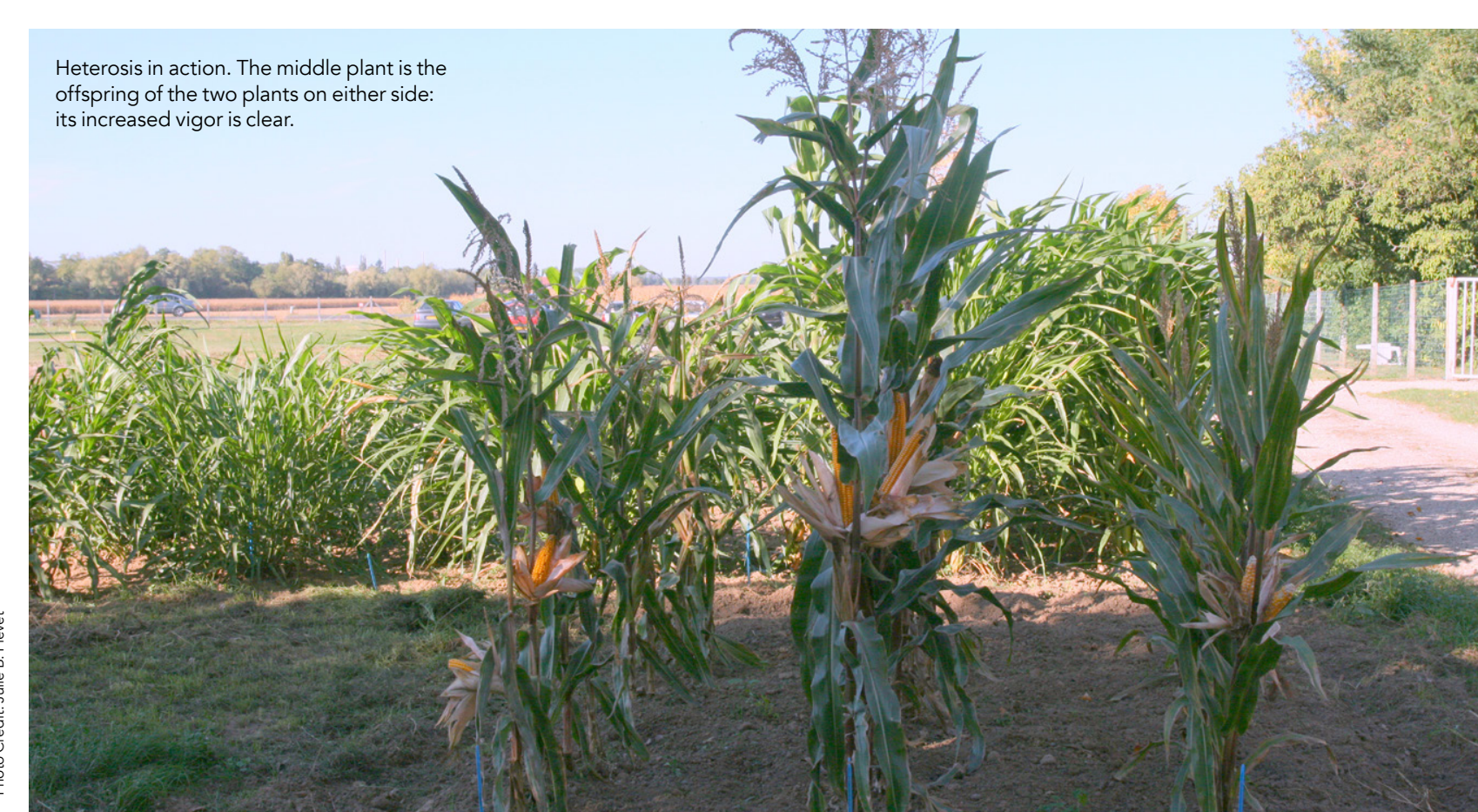

variants or single genes. Quantitative proteomics provides expression data for hundreds of proteins, including enzymes, while metabolomics techniques can access thousands of metabolites.

Complex information like this can be represented by networks to model the biological system of interest. Some of the most common
types of biological networks are proteinprotein interaction networks, metabolic networks, genetic interaction networks,
gene/transcriptional regulatory networks and cell signalling networks: heterosis could energe from all these networks.

\section{STUDYING GENOTYPE-PHENOTYPE} RELATIONSHIPS

The genetic makeup of an individual, its 'genotype', determines its characteristics or 'phenotype' in a given environment. The genotype-phenotype relationship is of fundamental interest to breeders as it describes how genetic polymorphism causes phenotypic variation. Genetic polymorphism due to mutations in the genotype produces the variety of forms

If the genotype-phenotype relationships were linear (proportionality between genotype and phenotype values), trait values compared to their parents, not better ones. Actually, the cellular processes involved in biological functions and structures are complex, and the relationship between measurable parameters at genotype and phenotype levels is often found to be non-linear. Network models increases, the metabolic flux (rate of synthesis of molecules catalysed by enzymes) through this network initially grows rapidly, as less often been investigated from $\begin{aligned} & \text { then slows down } \\ & \text { as the enzyme } \\ & \text { concentration goes }\end{aligned}$
the perspective of systems biology. as less often been investigated from $\begin{aligned} & \text { then slows down } \\ & \text { as the enzyme } \\ & \text { concentration goes }\end{aligned}$
the perspective of systems biology. in systems biology are typically highly to study phenotype responses to genotype variation.

Dominique de Vienne and colleagues suggest that heterosis is an emergent from non-linear relationships between genotypic variables and phenotypes or between different phenotypic levels, from 政 a systenic approach to show that the key "law of diminishing return

This 'law' states that in all productive processes, adding more of one factor
while holding all others constant, will (or rates) of biochemical and molecular related to enzyn

NON-LINEAR GENOTYPEPHENOTYPE RELATIONSHIP IS THE KEY OF HETEROSIS Non-linearity has been demonstrated at different levels of organisation, from cors explain the dominance of the most active allele over the least active, as proposed as early as 1934 by Sewall Wright, a fars

When the trait is controlled by many loci, which is the most common genetic transcription/translation to 


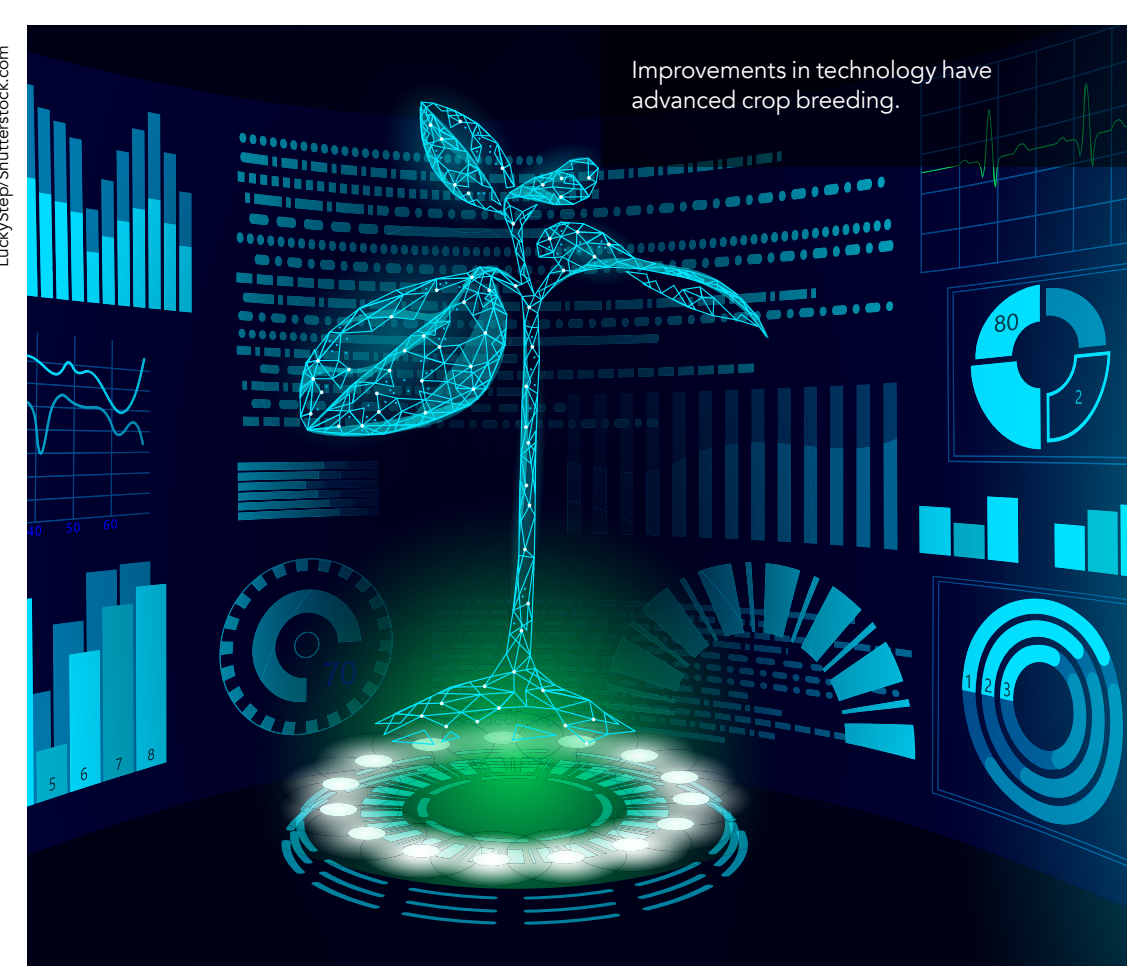

Mathematic modelling of physiological dominance suggested that heterosis is an intrinsic property of non-linear relationships between traits.

situation, heterosis arises as a look at metabolic flux prediction and First, the slightly deleterious recessive by sup of by other parent. The hybrid can therefore and so hybrid vigour is expected to be stronger when the parents are genetically distant due to better plementarity This model, likewise, explains inbreeding depression as the alleles at homozygous loci, i.e., loci with identical alleles. Second, the nonthat the effect of substituting one is dependent on the effect also plays a role in heterosis.

Dominique de Vienne and colleagues have mathematically formalised and validated the dominance/epistasis model of heterosis experimentally using in vitro and in sico (computer with the grolytic Thy have work assential to sim tate complex cellular behaviours from A simplified formalism based metabolic control andysis was used to derive global parameters that

four enzymes frem kinetic behaviour of of glycolysis. According to the structure of the pathway and the position of the enzyme in the pathway just one or two parameters per enzyme were sufficient.

Genetic variability was created by varying in silico enzyme concentrations. The virtual parents were crossed to computed. Again the curvature of the elationship describing the genotypephenotype relationship resulted in confis. This result is robust, as it was Whe

This mechanism for heterosis is valid eyond the metabolic systems. In Vominique de colleagues successfully predicted the amplitude of heterosis for two fitnessnumber - in series of hybrids among accessions of Arabidopsis thaliana, a valuable model plant for studies of growth and development and the first plant genome to be fully sequenced.

The traits of interest were non-linearly related to individual biomass, in the same way that metabolic fluxes are non-linearly related to enzyme concentrations. This non-linearity forces hybrids to deviate from the average to their better vigour Ma hematical up to $75 \%$ of the amplitude of heterosis while the genetic distance between parents explained at best $7 \%$ of heterosis.

Both mathematical and experimental results suggested that the appearance of heterosis in hybrids is a systemic property emerging from biologica complexity. These findings were consistent with various observations in and provide a model unifying the genetic effects underlying heterosis. The geometric view of genotype-phenotype for predicting heterosis in potentia for predicting heterosis in traits affecting quantitative and evolutionary genetics,

\section{Behind the Research}

Professor Dominique de Vienne

: dominique.de-vienne@inrae.fr T: + 33169332360

W: http://moulon.inrae.fr/personnes/ddevienne related traits - growth rate and fruit modelling made it possible to predict

\section{Detail}

Bio yeast. His publications include about 75 articles, 6 book scientists, 180 communications and posters in meetings,

\section{Co-authors} phenotype relationship, with special interest in genetics untangle the molecular bases of life-history traits in chapters, co-author of 3 textbooks for students and modelling efforts basses on con
Research Objectives

Prof de Vienne investigates the phenomenon of 'heterosis' or hybrid vigour.

Dominique de Vienne

Génétique quantitative et évolution-Le Moulon Ferme du Moulon
91190 Gif-sur-Yvette

Dominique de Vienne is Professor Emeritus, University Paris-Saclay, France and the former Director of the Quantitative Genetics and Evolution laboratory in Gif-sur-Yvette, France. His research area is genotypehe works on the bases of heterosis, on the evolution of enzyme concentrations and on the integration of proteomic data into constraint-based models to 41 invited international conferences, and 2 patents.

Funding

The work was supported by the French ANR programme grant from the French Ministère de la Jeunesse, 'Éducation Nationale et de la Recherche.

Julie B. Fiévet

Thibalt Nidelar

- Christine Dilmann

Prof de Vienne would also like to acknowledge Pr. André Gallais, his mentor in the field of quantitative genetics and heterosis.

\section{References}

Fiévet, J.B., Nidelet, T., Dillmann, C., de Vienne, D. (2018) genetics and computer simulations. Front. Genet 9, 159 Available at: https://doi.org/10.3389/fgene.2018.00159 Vasseur, F. et al. (2019) Nonlinear phenotypic variation uncovers the emergence of heterosis in Arabidopsis thaliana. PLoS Biology, 17(4), e3000214. Available at: https://doi.org/10.1371/journal.pbio.3000214

Fiévet, J.B., Dillmann, C., de Vienne, D. (2010) Systemic properties of metabolic networks lead to an epistasisGenetics, 120(2), 463-473.

Fiévet, J.B., Dillmann, C., Curien, G., de Vienne, D. (2006) Simplified modelling of metabolic pathways for reconstruction of the upper part of glycolysis. Biochemical Journal, 396, 317-326.

\section{Personal Response}

How has the field of plant genetics changed since

II Since I began working in plant genetics, there has the biologist. Robots for high-throughput genotyping and phenotyping, mass spectrometers for proteomics and metabolomics, increasingly powerful computers, etc., make it possible to accumulate and analyse huge possible to map finely and identify OTL for traits at all levels of biological organisation, from transcript/ and to better understand the genomic bases of phenotypic trait variation. For the breeder, markerselection methods to be implemented.

\section{universite PARIS-SACLAY} Heterosis is a systemic propent emerging from non-linear flux prediction and optimization: lessons from an in vitro

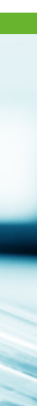

